Historically, the idea of how odours are detected was first suggested in the 1920s, but the concept of creating an electronic nose as a clever device consisting of a chemical-array sensor system for odour classification did not emerge until the early 1980s. Since then, this area of research has undergone rapid and tremendous development.

Electronic Noses is a fascinating and instructive book on the way our noses work, the progress made in the artificial detection and recognition of complex odours, and how this technology will be used in the near future. The authors' answer to the question, 'Why bother to try to recognize odours artificially?' is quite straightforward. First, from an academic point of view it is extremely challenging to try to figure out the biological and chemical mechanisms underlying our poorly understood sense of smell. Second, and more importantly, these olfactory machines are useful for routine industrial applications because, in contrast to humans, they are objective, reliable and do not get tired.

Although this is a technical account written mainly for an interdisciplinary scientific readership, the current and potential applications should appeal to anyone with interests from wine-tasting and food quality to environmental issues. This is a much-needed, clear and comprehensive review of the field of artificial olfaction as well as a basic treatment of the wide-ranging areas underlying its science and technology. As Julian Gardner and Philip Bartlett point out, our understanding of human odour detection and how we go about trying to mimic it coincides with a crucial time when instruments for doing this are starting to affect our everyday life.

After its introductory background, the book deals with the many requirements needed to make an actual device. The complex nature of the problem and the need for advanced technologies explains why the commercialization of these noses has only just begun. It is now possible to design devices capable of performing individual tasks 'similar' to our own olfactory system. This is a result of recent developments in microsensor technology, which have led to the creation of inexpensive integrated chemical sensors, together with applications specific to microprocessing devices and a better understanding of artificial intelligence.

Electronic Noses is not exactly an entertaining read. At face value, it is a collection and summary of research studies in the field, many of them from the authors' own work. However, it is much more than this in being, I believe, the first comprehensive and up-todate overview of the field.

Although the authors - a chemist and an engineer - appear to be on firmer ground when dealing with their own fields of expertise, they clearly and convincingly describe the functioning of our biological olfactory
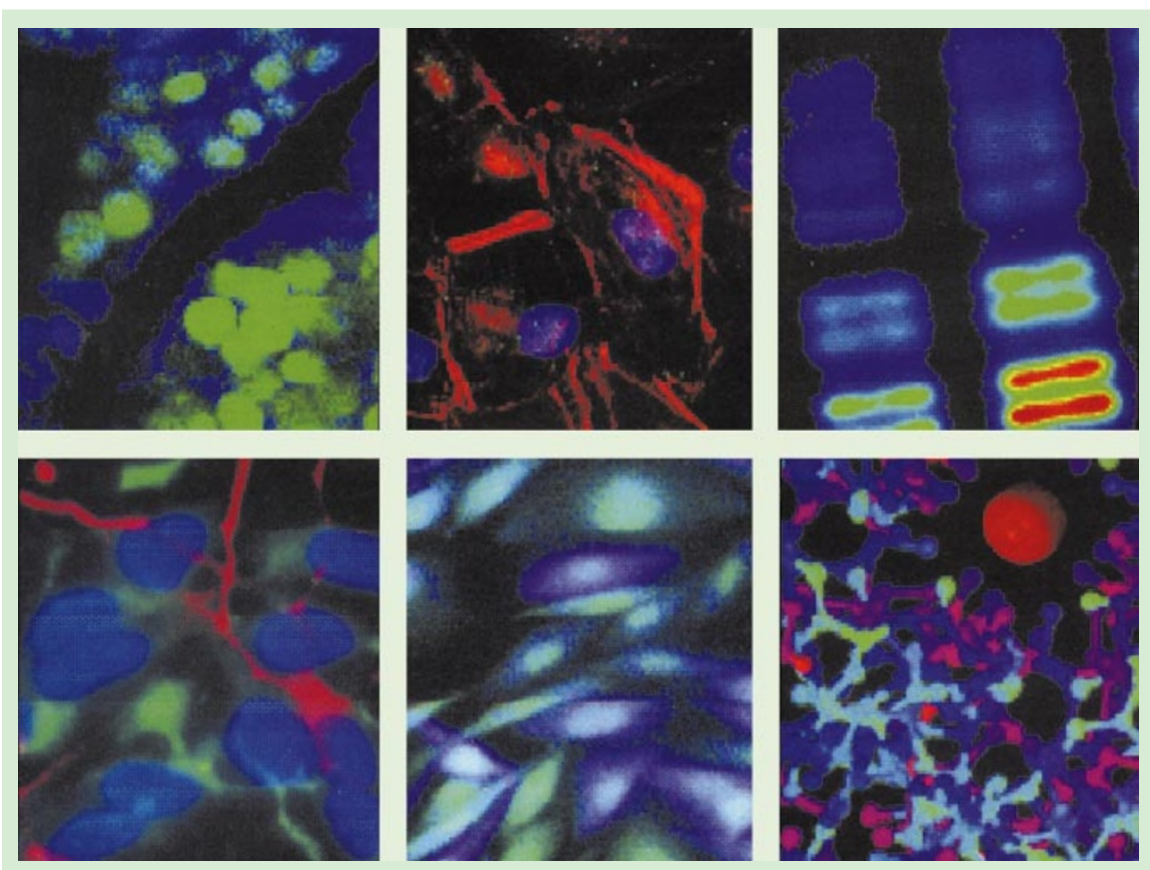

\title{
Cellular variations
}

Musically minded scientists Ira Melman, Richard Flavell and friends have turned to what they call biorock. The lyrics below are from one of the songs on their rock band's CD, Cellmates Volume 1 Number 1. (\$15, or $£ 12$ plus shipping. Apply to richard.flavell@yale.edu or ira.mellman@yale.edu)

“Kits-R-Us" (R. Flavell)

Way back in history they used to make up their ATP.

Pour out buckets of SSC, but screw that, suckers, that's not for me!

Now all that stuff just takes too much time, And it's my career that's on the line!

They cost some dough, let my PI whine Cuz I'm headed for the stars this time!

You buy it in one carton, it's got everything you need.

A few instructions to start on, can't fail to succeed.

processes for the non-specialist. I was disappointed that the historical and philosophical introduction to our sense of smell was not developed beyond the first two pages of the book, but, from the editors' perspective, it would perhaps have been beyond the scope of the book and the expertise of the authors.

But, if you are a gourmet and enjoy eating and drinking as much as I do, the chapter on the mechanisms of human perception of odour and flavour combined with the presentation of our biological olfactory machinery and its links with the brain will not only stimulate your intellect and olfactory neurons, but will also allow you to describe odours more precisely. You should, nevertheless, be warned that because of its technical aspects, Electronic Noses might well be difficult to digest in one go. Yet the final three case studies described, on the identification of perfumes, the freshness of fish and taints in beer, demonstrate the authors' desire to make
Dial 1-800-Kits-R-Us (free pipettes with every order)

Dial 1-800-Kits-R-Us (free pipettes with every order).

With just three tubes, red, white and blue, There's not too much for me to do.

Just mix it up, pipette that too, no need for fancy witch's brew.

And if perhaps the buffer's gone, just toss the kit, you can't go wrong!

You save so much time, science speeds along.

That's why I have time to write this song!

the book accessible to a wide readership.

The evolution of this technology depends on advances in many disciplines, and, as such, the future of the field is hard to predict. However, the potential market is so enormous and diverse that we can expect intense interest at least in the near future. Although our sense of smell has long been regarded as the least refined of our senses, and however confident the authors are about the rapid evolution of this technology, there is still a long way to go before such devices can accurately reproduce our subtle perception of flavours.

Many articles about this technology have appeared in the specialist literature, but it has not so far attracted much public attention. Hopefully, this comprehensive and thorough discussion of artificial olfaction and its fascinating interdisciplinary character will do just that.

Vincent Dusastre is an assistant editor at Nature. 